\section{CHRONIC KIDNEY DISEASE}

\section{AHR activation by uraemic solutes}

A new study reports activation of the aryl hydrocarbon receptor (AHR) in various tissues in models of chronic kidney disease (CKD) and acute kidney injury (AKI).

"AHR signalling is emerging as a mediator of uraemic toxicity, especially of indolic solutes, which are AHR ligands," says lead researcher Vipul Chitalia. "In this study we investigated the systemic activation of AHR, which may provide clues to the effects of uraemia on different organs."

To investigate AHR activation, the researchers used transgenic mice with a $\beta$-galactosidase reporter gene downstream of the DNA-binding domain for activated AHR. They report upregulation of $\beta$-galactosidase - indicating AHR activation - in the renal tubules, cardiac myocytes, hepatocytes and cerebral cortex microvasculature in mice with adenine-induced CKD and in mice with high plasma levels of indoxyl sulfate similar to those seen in end-stage renal disease. In both models, the levels of indoxyl sulfate correlated with $\beta$-galactosidase expression in the various tissues. "AHR activation in the central nervous system (CNS) suggests a role of uraemic solutes in neurocognitive deficiencies in CKD," comments Joshua Walker, first author of the study.

In mice with ischaemia-reperfusion injury, the plasma levels of indoxyl sulfate were increased at $24 \mathrm{~h}$ after surgery but then rapidly decreased, whereas the mRNA expression of $\beta$-galactosidase in the kidneys and liver, but not in the heart and CNS, was increased at $24 \mathrm{~h}$ and $48 \mathrm{~h}$ after surgery. The researchers suggest that this persistent AHR signalling following AKI might negatively affect the survival of the renal tubules.

"The results of our study are hypothesisgenerating; we believe they will seed future investigations into uraemic toxicity," says Chitalia. "First, knowing where AHR is active in various tissues and organs in response to uraemic solutes allows us to begin to examine which genes AHR regulates under these conditions. Second, from a clinical standpoint, our findings provide evidence to support the use of AHR inhibitors as therapeutics to combat complications that are associated with CKD and uraemia."

\section{Ellen F. Carney}

ORIGINAL ARTICLE Walker, J. A. et al. Temporal and tissuespecific activation of aryl hydrocarbon hydroxylase signaling may be visualized in discrete mouse models of kidney disease. Kidney Int. https://doi.org/10.1016/j.kint.2019.09.029 (2019)

\title{
Kidney miR-33 controls fatty acid oxidation
}

Renal fibrosis is a common pathological feature of chronic kidney disease and has been linked to the accumulation of lipids in the kidney. Now, Carlos FernándezHernando, Santiago Lamas and colleagues show that microRNA-33 (miR-33), known for regulating lipid metabolism, also affects the development of fibrosis in the kidney.

"Altered fatty acid oxidation (FAO) is important in kidney fibrosis and our previous work showed that miR-33 regulates FAO and cholesterol metabolism," explains Fernández-Hernando. In the folic acid-induced neprophathy (FAN) mouse model of kidney fibrosis, $M i r 33^{-/-}$mice had lower expression of fibrosis markers ( $\alpha$-smooth muscle actin, fibronectin and collagen) and lower levels of blood urea nitrogen (BUN) and creatinine than wild-type (WT) mice. Reconstituting the haematopoietic cell compartment of WT mice with Mir33-/- bone marrow did not improve fibrosis in the FAN model.

Oxygen consumption rate was enhanced in $\mathrm{Mir}^{33^{-/-}}$renal tubular epithelial cells but not in the presence of etomoxir, an inhibitor of carnitine palmitoyltransferase 1 (CPT1), which is an FAO regulator and a target of miR-33. In $\mathrm{Mir}^{33^{--}}$mice, CPT1A levels increase after FAN.

The researchers also used $\mathrm{pH}$ low insertion peptides ( $\mathrm{pHLIPs)}$ to deliver a miR-33 inhibitor to mice. pHLIPs deliver their cargo specifically to acidic environments and are known to accumulate in the kidney — in vitro, fluorescent $\mathrm{pHLIPs}$ accumulated in mouse primary renal tubular cells. In the FAN model, mice treated with anti-miR-33 ${ }^{\text {pHLIP }}$ had higher CPT1A, improved kidney fibrosis, and lower BUN and creatinine levels compared with untreated mice. "miR-33 regulates lipid metabolism in the pathological setting of kidney fibrosis," concludes Lamas.

"Next, we want to explore how different miR-33 targets contribute to kidney fibrosis," explains Fernández-Hernando. "Selective microRNA inhibition in the kidney with the pHLIP system should also be explored for therapeutic use," adds Lamas.

Monica Wang

ORIGINAL ARTICLE Price, N. L. et al. Genetic deficiency or pharmacological inhibition of miR-33 protects from kidney fibrosis. JCl Insight https://doi.org/10.1172/jci.insight.131102 (2019)

\section{HYPERTENSION}

\section{Sex-dependent effects of ERAP1 deficiency}

The renin-angiotensin system regulates blood pressure (BP) and mounting evidence indicates that biological sex affects the pathways activated by these hormonal mediators. Now, Jose Romero and colleagues report a sex bias on the effects of endoplasmic reticulum aminopeptidase 1 (ERAP1) deficiency.

"We had previously reported an effect of biological sex on systolic BP responses to angiotensin II (Angll), a potent vasoconstrictor and inducer of aldosterone secretion from the adrenal gland," explains Romero. "We then focused our studies on ERAP1, which had been shown to degrade Angll in vitro."

"In female and male mice, ERAP1 deficiency increased tissue Angll - systolic BP, diastolic $\mathrm{BP}$ and salt sensitivity of BP were also increased, indicating that loss of ERAP1 leads to volume expansion," remarks Romero. However, despite similar increases in BP, aldosterone levels increased in Erap1 ${ }^{+/-}$males but not in females. Volume expansion would be expected to increase renal plasma flow, but this physiological response was only observed in male Erap1 ${ }^{+/-}$mice; glomerular volume also only increased in Erap ${ }^{+/-}$males. Therefore, despite an increase in BP, ERAP1 deficiency did not induce renovascular changes in female mice. In humans, homozygosity for the ERAP1 rs30187 loss-of-function polymorphism was significantly associated with higher BP than no expression of the risk allele or heterozygosity - this effect was only observed in males, not females. "Our results suggest that men homozygous for the rs30187 risk allele might benefit more from therapeutic Angll receptor blockade or angiotensin-converting enzyme (ACE) inhibition than women," notes Romero.

"Our study provides a rationale for clinical trials that specifically assess the effects of biological sex on BP control through Angll blockade, ACE inhibition and mineralocorticoid receptor antagonism," concludes Romero.

Monica Wang

ORIGINAL ARTICLE Ranjit, S. et al. Sex-specific differences in endoplasmic reticulum aminopeptidase 1 modulation influence blood pressure and renin-angiotensin system responses. JCl Insight 4, e129615 (2019) 